\title{
III.
}

\section{Neue Bemerkungen über die Pistoieser Glosse zum Justinianischen Codex.}

Von

\section{Herrn Avv. Luigi Chiappelli,}

Privatdocenten der Rechte an der K. Universität zu Pisa. ${ }^{*}$ )

Im 2. Hefte der Romanistischen Abtheilung des 7. Bandes dieser Zeitschrift S. 2 ff. (1886) hat Herr Prof. Hermann Fitting die Ausgabe und Besprechung der in der Ueberschrift genannten Glosse in meiner Schrift „La glossa pistoiese al Codice Giustinianeo" (Memorie della R. Accademia di Scienze di Torino. Torino, Loescher, 1885) in eingehender und wohlwollender Weise angezeigt und mich dabei zur Zusammenstellung eines Verzeichnisses der in der Handschrift enthaltenen jüngeren Glossen aufgefordert, um mit grösserer Sicherheit über den geschichtlichen Werth der Glosse und über den Stand der Wissenschaft des römischen Rechtes in den verschiedenen Jahrhunderten des Mittelalters urtheilen zu können. ${ }^{1}$ )

*) Mit dem Auftrage zur Uebersetzung dieser Abhandlung hat mir der Herr Verfasser zugleich die Erlaubniss gegeben, nach Ermessen eigene Zusätze zu machen. Ich habe von dieser Erlaubniss einige Male in den Anmerkungen, jedesmal mit Beifügung meines Namens, Gebrauch gemacht.

Fitting.

1) Auch in der Kritischen Vierteljahresschrift f. Gesetzgebung und Rechtswissensehaft Bd. 28, Heft 2, S. 220 ff. (1886) ist über meine Ausgabe der Pistoieser Glosse eine Besprechung von Herrn Prof. Krüger erschienen, welcher mit Recht eine Unterscheidung der verschiedenen Schreiber fordert, von denen die Glossen herrühren. Ich bin auch darin vollkommen mit Herrn Krüger einverstanden, dass es zur Ermöglichung eines gründlichen Urtheils über das Alter der älteren Glossen nothwendig sein würde zu untersuchen, ob sie aus früherer Zeit stammen als die Arbeit der Ergänzung der alten Epitome des Codex. Allein zu dieser Untersuchung wäre zweierlei unerlässlich gewesen. Vor allem die Kenntniss aller epitomirten Handschriften des Codex, wozu ich nicht in der Lage war; meine Arbeit bezweckte nur 
Diesem sachkundigen Rathe mit lebhaftem Danke folgend, mache ich es mir zur angenehmen Ptlicht, den Gelehrten eine chronologische Uebersicht der verschiedenen Glossen vorzulegen, welche in ihrer Gesammtheit die sog. Pistoieser Glosse bilden.

Uebrigens muss ich, bevor ich weiter gehe, sagen, dass ich bei erneuter Prüfung der Pistoieser Codexhandschrift (Arcbiv des Pistoieser Domcapitels Nr. 66) darin mittels chemischer Reagentien noch andere, ursprünglich nicht lesbare Glossen habe entdecken können. Ich habe in der folgenden Uebersicht diese neuen Glossen zusammengestellt. Sie beziehen sich auf die am Rande der Handschrift eingeschalteten Constitutionen des Codex, Ergänzungen, welche zum Theil von dem ursprünglichen und ältesten Schreiber des Textes, zum Theil von späteren Händen herrühren. Ich veröffentliche diese Glossen nach den von mir bei der Ausgabe der Pistoieser Glosse befolgten Grundsätzen und mit Fort-

einen Beitrag zu einer weitergehenden, von welcher es erwünscht wäre, wenn sie Herr Krüger selbst mit seiner bewährten Sachkunde in Angriff nähme. Zweitens wäre es nothwendig gewesen, den Umfang der gedachten Epitome zu kennen, deren Inhalt Herr Krŭger bloss für die vier ersten Bücher des Codex angegeben hat. Das hätte mich aber zu einer neuen Arbeit mit anderen Zwecken geführt. - Herr Krüger macht mir auclı einige Missverständnisse des Textes der Glosse zum Vorwurfe. Während ich ihm in einigen Punkten zustimme, muss ich in Ansehung der übrigen sagen, dass meine Ausgabe die Handschrift genau wiedergibt. Uebrigens sind manche der von ihm hervorgehobenen Irrthümer nicht bedeutend genug, um den Text zu trüben, und leicht vom Leser zu verbessern. Nicht wenige davon sind auch Druckfehler, wie die willkürliche Zusammenziehung von Wörtern des Textes in einer Anzahl von Glossen. Andere solche Zusammenziehungen, wenn auch sonderbar, finden sich wirklich in der Pistoieser Handschrift des Codex, Auch ist es nicht genau, wenn er sagt, ich habe angenommen, dass diese Handschrift das Werk eines einzigen Schreibers sei; dies erhellt sowohl aus meiner Ausgabe der Pistoieser Glosse als aus meiner anderen Abhandlung: „Nuovo Esame del manoscritto pistoiese del Codice Giustinianeo. Roma 1885". Ich glaube, dass der Text der Handschrift im ganzen von einem und demselben Schreiber herrührt, aber dass viele Einschaltungen von Constitutionen am Rande und die in die Handschrift eingelegten kleinen Pergamentstreifen von acht anderen Abschreibern herrühren, denen übrigens auch sehr viele Glossen zuzutheilen sind. 
führung der in dieser Ausgabe auftretenden Zählung, um die bequeme Citirung auch dieser Glossen zu ermöglichen.

Scholien (Scolii).

$n^{\circ} .926 \ldots$ Lib. III. tit. XXXII. const. 26. -

Nota . mora litis .. post contestationem - non (?)

po ..t s..... p..d ..e (prodesse?) non potest. ${ }^{\text {) }}$ )

Kritische Glossen (Glosse critiche).

no. 927 ... Lib. I. tit. LI. const. 14 pr. -

v. quibus res publica . . . . . alias. cui. ${ }^{2}$ )

$n^{\circ} .928 \ldots$ Lib. II. tit. IV. const. 5. -. v.

legitimam aetatem . . . . . fecisti.

$n^{\text {o. }} 929$... Lib. II. tit. XX. (XIX. ed. Krüger.)

const. 5. - v. vero sciente . . . . emptore.

$n^{\circ} .930$... L Lib. II. tit. LII. const. 7 pr. -

v. crimen (discrimen.) . . . . . . discrimen.

no. 931 ... Lib. IlI. tit. XXXI. const. 3. -

v. Si + materterae . . . . . . . hereditas.

$n^{\circ} .932 \ldots$ Lib. IV. tit. XXVII. const. 2 (al. 3)

$\S 1$. - v. personali . . . . . . . in.

$\mathrm{n}^{\circ} .933 \ldots$ Lib. V. tit. XI. const. 4. -. v.

pactum esse. . . . . . . . petentis.

no. 934 ... Lib. V. tit. LI. const. 8. 一. v. si is (his) . . . . . . . . . . his.

no. 935 ... Lib. V. tit. LI. const. 8. -. v. acceptilatione . . . . . . . . . uel nouatione.

$n^{\circ} .936 \ldots$ Lib. VI. tit. LXI. const. 8. $\S 1$ in

f. -. v. restitutionis + minime . . auxilio.

$n^{\circ} .937 \ldots$ Lib. VII. tit. LIII. const. 9. -.

v. $\operatorname{Eos}$

1) Die Schrift dieses Scholion ist selır schadhaft, so dass die Farbe der Tinte verscliwunden und die Glosse auf den Pergamente kaum sichtbar ist.

2) Zufolge Druckfehlers ist in meiner Angabe des Inhaltes der Pistoieser Codexhandschrift (Nuovo Esame del manoscritto pistoiese p. 9) die Const. 14 tit. 51, welche in der Handschrift steht, ausgelassen. 
Erklärende Glossen (Glosse interpretative). $n^{\circ} .938$. . . Lib. II. tit. LII. const. 7.

$\S 2$. - . v. aenumeratae . . si propter officium. $n^{\circ} .939$. . . Lib. III. tit, VIII. const. 2.

-. v. si de ea re . . . . scilicet . de genere. no. $940 \ldots$ Lib. IV. tit. I. const. 5. v. efficiet.

$n^{\circ} .941 \ldots$ Lib. IV, tit. VI. const. 7. -. v. leuitati. . . . . scilicet f fallatiae,

$n^{\circ} .942 \ldots$ Lib. IV. tit. XXVI. const.

10 in f. - - v. mobiles ementes de seruo. no. $943 \ldots$ Lib. IV. tit. XXIX. const.

2. - v. intercessionibus . . id est. obligationibus. $n^{\circ} .944 \ldots$ Lib. 1V. tit. XXIX. const.

2. 一. v. ab eo . . . . . scilicet , creditore.

In Ansehung dieser neuen Glossen können die Bemerkungen wiederholt werden, welche ich bereits in der Ausgabe der Pistoieser Glosse gemacht habe, da die kenuzeichnenden Eigenschaften der letzteren auch bei ihnen anzutreffen sind. Daher gehe ich hierüber kurz hinweg und wende mich zum hauptsächlichsten Gegenstande dieser Arbeit.

Um über die Zeit, zu welcher die verschiedenen Glossen verfasst worden sind, mit Sicherheit zu urtheilen, genügt es meines Erachtens nicht, genau zu unterscheiden; was jedem der Schreiber und Bearbeiter der Pistoieser Handschrift zuzutheilen ist; man muss auch die Glossen, welche sich auf den alten Kern des Textes dieser Handschrift beziehen, von denjenigen unterscheiden, welche zu den später am Rande behufs der Ergänzung des epitomirten Codex eingeschalteten Constitutionen gehören. Dies verschafft natürlich Licht über die ursprüngliche Ausdehnung der Glosse.

Welches sind nun die Glossen, welche sich ausschliesslich auf die ursprüngliche Epitome des Codex beziehen und mithin älter sind als die Arbeit jener Ergänzung dès Textes? In der Pistoieser Glosse zeigen sich einige Scholien mit Bestimmtheit als ursprüngliche, die kritische Arbeit der Vervollständigung der alten Epitome an Alter übertreffende Erläuterungen; daneben treten aber auch viele Glossen auf, 
welche gleichen Alters mit dieser Arbeit oder jünger sind. Eine genaue Unterscheidung der ursprünglichen, auf die Bestandtheile der alten Epitome selbst sich beziehenden Glossen ist sehr schwierig und würde eine weitschichtige Arbeit elheischen, welche die Grenzen unserer Aufgabe überschreiten würde; es würde nämlich nothwendig sein, die Wiederherstellung der ursprünglichen Form der Epitome des Codex vollständig durchzuführen, wie sie für die vier ersten Bücher bereits von Krüger geschehen ist ${ }^{1}$ ). Wenn ich mich demnach auf dieses schwierige, gereiftere Kraft und Erfahrung erfordernde Unternehmen nicht einlassen kann, so halte ich es doch für sicher, dass die Unterscheidung der Glossen zum Texte von denjenigen zu den eingeschalteten Constitutionen für die angedeutete Untersuchung ein nützliches Hülfsmittel sein wird. Man beachte dabei immer, dass diese Zusätze zu dem epitomirten Codex am Rande theils von dem Schreiber des ursprünglichen Textes selbst gemacht sind, theils von späteren Schreibern bis zum 12. Jahrhundert. Im folgenden sind die Glossen zu den auf den Rand unserer Handschrift geschriebenen Constitutionen zusammengestellt:

Nr. 26. 182. 201. 222. 284. 413. 571. 647. 648. 649. 669. 670. 671. 747. 926. 927. 928. 929. 930. 931. 932. 933. 934. 935. 936. 937. 938. 939. 940. 941. 942. 943. 944.

Unterwirft man nun die sämmtlichen in der Pistoieser Codexhandschrift enthaltenen Glossen einer aufmerksamen Prüfung, so wird man zu dem Schlusse geführt, lass abgesehen von dem ursprünglichen Grundstocke derselben, welcher ohne Vergleich umfangreicher als die nachträglichen Zuthaten ist, sich darin Glossen finden, welche immerhin auch noch dem 10. Jahrhundert angehören, aber von zwei neuen Händen geschrieben sind. Sodann finden sich Glossen, deren Alter ungewiss bleibt und zwischen dem 10. und 11. Jahrhundert schwankt. Andere Glossen, und zwar recht zahlreiche, ge-

1) Codex Justinianus recens. P. Krüger. Berol. 1877. App. II. p. * 58. In Ansehung des Anfangs des fünften Buches der Epitome s. Krüger, Ueber d. Epit. u. d. Subscript. im vierten Buch d. Just. Cod. (Zeitschr. f. Rechtsgesch. VIII. S. 25). 
hören zweifellos dem 11. Jahrhundert an. Hienach unterbreiten wir, mit Ausschluss der von dem ursprünglichen Schreiber herrührenden Glossen, der Aufmerksamkeit derjenigen, welche für dieses alte Rechtsdenkmal ein Interesse haben, in dem nachstehenden dreifachen Verzeichnisse die Glossen in chronologischer Uebersicht.

Glossen des 10. Jahrhunderts (Spätere Hände).

Nr. 135. 220. 251. 261. 268. 274. 275. 323. 368. 381. 398. 407. 428. 438. 484. 485. 543. 544. 545. 647. 648. 649. 683, 684. 705. 706. 775. 776. 831. 834. 835 . 836. 897. 932. 936 .

Glossen ungewissen Alters (10. oder 11. Jahrhundert).

Nr. 20. 26. 34. 85. 104. 183. 184. 185. 186. 188. 189. 190. 191. 192. 193. 194. 196. 199. 200. 203. 207. 210. 217. 219. 221. 222. 223. 227. 237. 669. 670 . 671. 674. 679. 680. 681. 682, 685. 792. 798. 927. 930. 938 .

Glossen des 11. Jahrhunderts.

Nr. 9. 11. 15. 16. 19. 27. 29. 30. 3!. 32. 35. 46. 47. 48. 51. 52. 75. 78. 83. 86. 87. 92. 94. 100. 105. 106. 125. 126. 128. 129. 130. 136. 137. 142. 143. 144. 145. 148. 155. 157. 158. 160. 161. 162. 165. 173. 174. 175. 176. 178. 181. 182. 213. 218. 224. 229. 230. 232. 233. 234. 235. 236. 238. 239. 240. 241. 242. 245. 257. 260. 265. 273. 278. 284. 303. 312. 313. 340. 365. 366. 369. 383. 414. 422. 446. 461. 463. 464. 477. 482. 491. 504. 508. 533. 539. 565. 569. 571. 577. 578. 597. 613, 619. 627. 630. 645 . 650. 658. 659. 662. 693. 786. 840. 841. 851. 852. 857. 859. 865. 888. 894. 931.937.939.1)

1) Bereits Fitting in der Eingangs erwähnten Anzeige S. 12 hatte die Vermuthung ausgesprochen, dass die Glossen 16, 19, 20, 34, 56, $100,775,776,792,798,897$ nicht zu dem ursprünglichen Glossenbestande gehören möchten. Die in dieser Reihe von Fitting genannte Glosse 56 starnmt jedoch von dem ursprünglichen Schreiber des Textes. 
Ausser den in diese Klassen eingereihten und den zu dem alten Grundstocke gehörenden Glossen sind auf den beiden ersten Seiten der Handschrift die Spuren von vier jetzt unentzifferbaren, mit grünlicher. Tinte geschriebenen Glossen bemerkbar, welçhe den const. 1 und 4 tit. I. und der const. 1 tit. II. des ersten Buches beigesetzt sind. Soweit die wenigen lesbaren Buchstaben, die Farbe der Tinte und die Gestalt der Schrift einen Schluss gestatten, haben diese Glossen den nämlichen Urheber wie einige Glossen zu dem Texte des alten Statutes von Pistoia. Die Handschrift desselben wird ebenfalls in dem Archiv des Domcapitels zu Pistoia aufbewahrt und ist mit Nr. 40 bezeichnet. Dieses Statut wird schon von den alten Geschichtschreibern von Pistoia ${ }^{1}$, dann von Zaccaria, Muratori und Berlan ${ }^{2}$ ), welche es nach einander veröffentlicht haben, dem Jahr 1107 oder allerspätestens dem Jahr 1117 zugewiesen. Diese Meinung stiutzte sich auf die folgende Lesung der Ueberschrift des Statutes:

"In nomine sancte et indiuidue trinitatis. Amen. Anno ab eius natiuitate. millesimo. contesimo. septimo. Indictione. XI. VI. Kal. Decembris. Ad honorem dei et domini Henrici Romanorum imperatoris."

In einer Arbeit, welche soeben im Archivio Storico Italiano veröffentlicht wird, haben wir diese Lesung auf Grund einer genauen Untersuchung der Handschrift folgendermassen verbessert:

„In nomine sancte et indiuidue trinitatis. Amen. Anno ab eius natiuitate. millesimo. centesimo. septimo. Indictione XI. VI. Kal. Decembris. Ad honorem dei et ctomini frederici Romanorum imperatoris."

Auch Schlüsse aus dem Inhalte des Statutes, worin Ereignisse aus der zweiten Hälfte des 12. Jahrhunderts erwähnt

1) Salvi - Historie di Pistoia. 1656. v. 1. p. 58. Fioravanti Memorie storictre della cittì di Pjstoia. 1758. p. 167, e seg.

2) Zaccaria - Anecdota Medii $\Lambda$ evi. p. 217. - Muratori - Anli(. Stat. Med. Nevi. v. 4. col. 527. - Berlan - Studi storico critici sugli Statuti Pistoiesi. Pistoia. 1874. Prefaz. - Berlan - Statuti di Pistoia del secolo XII. Bologna. 1882. p. 113. - Sclopis - Storia della legislazione italiana. Pariyi. 1861. v. 2. cap. V. Pertile - Sturia del dirit to italiano. v. 2. p. 2. pag. 658, c seg. 
sind, führen uns zu: der Annahme, dass nicht die Angabe der Indiction auf Irrthum beruht, sondern dass aus Uuiachtsamkeit des Abschreibers das Wort "septuagesimo" bei der Angabe der Jahreszahl ausgelassen ist. Wir sind demnach zu der Folgerung gelangt, dass dieses Statut dem Jahr 1177 angehört, dass die späteren Abünderungen desselben bis 1183 herabreichen, und dass ihre Glossen in den letzten Jahren des 12. Jahrhunderts verfasst sind. ${ }^{1}$ )

Dieses Ergebniss ist auch für die gegenwärtige Untersuchung von Nutzen, weil es die Annahme begründet, dass die Pistoieser Handschrift des Codex mehrere Jahrhunderte hindurch der Gegenstand des Studiums gewesen ist, und dass ihre Glossen einen Zeitraum umfassen, welcher sich mindestens. vom 10. bis zum 13. Jahrhundert erstreckt. Sie schliesst also eine walure Geschichte des Betriebes des römischen Rechtes während einer langen Zeit des Mittelalters in sich ein, und auch hierin beriihrt sie sich mit der alten Turiner Institutionenglosse. Auch aus dieser Thatsache, dass unser Glossenwerk vom frühesten Mittelalter bis zu der glorreichen Zeit der Bologneser Schule herabgeht, darf man mit Sicherheit schliessen, dass die wissenschaftliche Arbeit dieser beiden Zeitalter durch ein inniges Band verknüpft ist, was bis jetzt zu den dunkelsten Punkten der Geschichte der Rechtswissenschaft im Mittelalter gehört. Die leicht zu vermuthende ununterbrochene Fortdauer der wissenschaftlichen Ueberlieferung erhält so eine nicht werthlose Bestätigung. Dabei ist auch der Umstand bemerkenswerth, dass die Darmstädter Handschrift Nr. 2000, welche, wie wir in der Ausgabe der Pistoieser Glosse gezcigt haben, die letztere $z u$ einem grossen Theil enthält, durch einander sowohl Glossen des ältesten Grundstockes aufweist, als solche, welche dem 11. Jahrhundert angehören. Das nämliche lässt sich auch von der Pariser

1) Chiappelli, Età degli antichissimi Statuti di Pistoia. (Estr. dall' Archivio Storico Italiano.) Man findet in dieser Abhandlung die nähere Begründung der Behauptung, dass dieses Statut während der Regierung des Kaisers Friedrich Barbarossa, und gerale in Jahr 1177 erlassen ist. Das letzte in der Handschrift enthaltene Statut ist von 1183 datirt. 
Handschrift Nr. 4516 sagen im Hinblick auf das, was Krüger in der (S. 86 Anm. 1) gedachten Besprechung berichtet.

Ist es sonach richtig, dass, wie wir vorhin gesagt haben, unser Glossenwerk, zu dessen Bildung Männer aus mehreren Jahrhunderten beigetragen haben, eine Geschichte des Betriebes des römischen Rechtes während eines langen Zeitraumes des Mittelalters einschliesst, so ist es nothwendig, nach der Eintheilung sämmtlicher Glossen in zeitliche Gruppen die hervorstechendsten Merkmale einer jeden dieser Gruppen zu untersuchen. Zufolge dieser Untersuchung wird es möglich, bis zu einem gewissen Grade das Mass der Bildung der verschiedenen Glossenverfasser sowie des Zeitalters, welchem jeder von ihnen angehörte, zu unterscheiden.

Es ist jetzt durch meine Ausgabe der Pistoieser Glosse und durch die bisherigen Erörterungen gewiss, dass der Hauptbestand der in ihr entlaltenen einzelnen Glossen dem ursprünglichen Schreiber der Pistoieser Codexhandschrift zugeschrieben werden muss. In den Glossen dieser ersten Klasse, welche im 10. Jahrhundert abgeschrieben worden sind, aber - wenigstens zu einem grossen Theil — sowohl nach Fitting's als nach meiner Ansicht aus einer noch weit früheren Zeit herstammen, herrschen die Scholien vor, welche die in den einzelnen Gesetzesstellen enthaltenen allgemeineren Regeln aufzuweisen bestimmt sind und als Vorläufer der späteren Brocarda erscheinen. Mit Ausnahme des Schol. 926 sind sie den Constitutionen beigesetzt, welche den Grundstock der Handschrift ausmachen, und nicht den am Rande eingeschalteten. Auch treten unter ihnen in grosser Zahl die grammatikalischen Glossen auf, welche es bestätigen, dass das Rechtsstudium in jenen frühen Zeiten in den Schulen betrieben wurde, in denen man Grammatik, Dialektik und Rhetorik lehrte. Nicht minder kommen darunter zahlreiche textkritische Glossen vor. Quellen dieser ältesten Glossengruppe sind der Codex selbst mit Ausnahme der letzten drei Bücher, und die Epitome Juliani (gl. 97, 856). Die Form anlangend, verdienen Beachtung die Ausdrücke: ,c a pitula" im Sinn von Gesetzen ${ }^{1}$ ), „corrumpi“" zur Bezeichnung von Aenderungen

2) Dieser Ausdruck findet sich in der Glosse 82 und ist bekanntermassen den Juristen zu Pavia und der juristischen Literatur des 
von Gesetzen ${ }^{1}$ ), ,, interessi es als Substantivum ${ }^{2}$ ) und „placitum $^{\text {" }}$ in der Bedeutung von iudicium ${ }^{3}$ ): vier Ausdrücke, welche zwischen diesen.Glossen und anderen Arbeiten späterer Jahrhunderte, besonders des 11. Jahrhunderts, eine Bezichung herstellen. Uebrigens weist diese erste Masse von Glossen ein hohes Alter auf.

11. Jahrhunderts eigen. Fitting sagt. in der Eingangs genannten Anzeige S. 6, diese Citirform sei von der Bologneser Schule nicht gebraucht worden; dagegen beziehe ich mich aber, um nicht von anderen Beispielen zu reden, auf die glossa 1 von Irnerius, welche ich in folgender Schrift herausgegeben habe: „Ghiappelli, Glosse d' Irnerio e della sua scuola“. (Mem. della R. Accademia dei Lincei. Roma. 1886. p. 20.)

[Der letzten Bemerkung dürfte doch wohl ein Missverständniss zu Grunde liegen. Meine Behauptung an dem von Chiappelli angefïhrten Orte bezog sich hloss auf Citate von Stellen des Codex, und wenn ich auch anderwärts dieselbe Behauptung wohl in allgemeinerer Weise gemacht habe, so waren doch in der That überall nur Citate aus dem Godex und den Digesten gemeint. Vergl. meine Ausgabe der Glosse zu den Exceptiones L. R. des Petrus S. 20. Dass aber die Stellen des Codex und der Digesten von der Bologneser Schule nirgends als ,capitula", sondern überall als "leges" citirt werden, wird durch die von Chiappelli veröffentlichten Glossen des Irnerius und seiner Nachfolger nicht widerlegt, sondern vollauf bestätigt. In der Glosse des Irnerius, worauf sich Chiappelli beruft, und ebenso in der glo. 27 des Placentimus, welche er in seiner oben von ihm genannten Schrift S. 14 ebenfalls als Beleg für den Gebrauch des Ausdruckes „capitula“ in Citaten der Glossatorenschule nennt, handelt es sich um Verweisungen nicht auf den Codex oder die Digesten, sondern auf Capitel von Novellen, und zwar in der Glosse des Irnerius nicht, wie Chiappelli meint, um ein falsch bezeichnetes Citat des cap. 25 der Nov. 22 nach dem Autheníicum, sondern, was sehr bemerkenswerth, um das ganz richtige Gitat der Rubrik des cap. 31 der dieser Novelle entsprechenden const. XXXVI. der Epitome Iuliani. Dass diese Capitel so, wie im Gesetzestexte selbst und demzufolge in Citaten aus heutiger Zeit, auch in Citaten der Bologneser Schule als „capitula" auftreten, hat doch gewiss nichts auffallendes und ist schwerlich geeignet, gegen meine hier zur Frage stehende Behauptung einen Gegenbeweis zu liefern. Fitting.]

1) Siehe die Glossen 97, 8506. Fitting in der erwähnten Anzeige weist auf die verwandte Form "rumpi" in der Expositio zum Liber Papiensis hin; es ist darin aber auch geradezu der Ausdruck ,corrumpi" in der nämlichen Bedeutung anzutreffen. Siehe Expos. ad Rothar. $141 \S 3,163 \S 1$.

2) Siehe Glosse 80 .

3) Siehe Glosse 725 . 
Die zweite Masse, von zwei späteren Schreibern des 10. Jahrhùnderts herrührend, enthält wenige Scholien und viele kritische Glossen. Die grammatikalische Erläuterung zeigt die Neigung zu verschwinden, und diejenigen, welche sich mit dem Codex beschäftigen, fühlen lebhaft das Bedürfniss, den Gesetzestext zu ergänzen und zu verbessern, sowie sich andere Quellen zu Nutze zu machen. In der That finden sich in diesen Glossen die Spuren der Benutzung der Institutionen ${ }^{1}$ ) und der Werke Isidor's für die Definition von invasio ${ }^{2}$ ).

Zwischen dieser zweiten Gruppe und der dritten haben wir eine Anzahl von Glossen aus ungewisser, zwischen dem 10. und dem 11. Jahrhundert schwankender Zeit unterschieden. Darunter sind zweie von höchster Bedeutung, nämlich die Glossen 20 und 792, in welchen der Einfluss der Digesten zu bemerken ist; doch ist es sicher, dass die hier von dem Verfasser der Glossen bekundete Kenntniss der Digesten keine unmittelbare ist. ${ }^{3}$ ) Dagegen sind nichtjuristische Quellen benutzt: die Werke Alcuin's für die Definition von narratio ${ }^{4}$ ) und diejenigen Isidor's für die Definition von chi rog raphu m5). Diese Definitionen verknüpfen die gedachten Glossen mit dem Literaturkreise der Exceptiones des Petrus, der Epitome Exactis regibus und des Libellus de Verbis legalibus. Auch die, wenngleich nur mittelbare, Benutzung der Digesten befestigt die Ansicht, dass diese Glossen entweder dem Ende des 10. oder rlem Anfange des 11. Jahrhunderts angehören.

9) Siehe Glossen 775,776 .

2) Siehe Glosse 897 .

3) [Ich kann mich den Ausführungen Chiappelli's hier nicht überall anschliessen. Glosse 20 ist wörtlich aus Isidor (Orig. V, 26) entnommen and kann daher meines Erachtens zur Beantwortung der Frage, ob dem Urheber die Digesten bekannt waren, überhaupt nichts beitragen. Dagegen gibt Glosse 792 die erste Hälfte der I. 6 Dig. de reb. cred. 12, 1 so wörtlich wieder, dass es doch allermindestens zweifelhaft sein kann, ob sie nicht unnittelbar aus den Digesten geschöpft sei. Fitting.]

4) Siehe Glosse 34. [Hier könnte aber auch ummittelbar aus Gicero (De invent. I. c. 19) gesehöpft sein. Fitting.]

5) Siehe Glosse 798. 
In der dritten Gruppe von Glossen, welche ich dem 11. Jahrhundert zuschreibe, sind nicht wenige Scholien und textkritische Glossen enthalten. Man begegnet auch noch den Spuren der grammatikalischen Erläuterung, aber nur als letzten Ausläufern einer alten, ihrem Erlöschen nahen Ueberlieferung. Dasjenige, was die hauptsächlichste Eigenthümlichkeit dieser Masse von Glossen ausmacht, gibt eine Bestätigung dessen, was wir in Ansehung ihres Alters gesagt haben. In diesen Glossen ist nämlich besonders bemerkenswerth die Menge der Definitionen, welche ein charakteristischer Zug der juristischen Literatur des 11. Jahrhunderts ist. Ein Theil dieser Definitionen ist aus Isidor genommen ${ }^{1}$ ), ein anderer Theil findet sich mit geringen Abweichungen in anderen gleichzeitigen ${ }^{2}$ ) oder wenig späteren Schriften wieder, wie in den Exceptiones des Petrus und ihrer Glosse, im Libellus de Verbis legalibus, im Liber iuris Florentinus, im Brachylogus und in der Kölner Institutionenglosse. Dic Hülfsquellen dieser Glossengruppe sind eine lateinische Uebersetzung der griechischen const. 4 Cod. VI, $4^{3}$ ) und die Werke des Isidor; auch in ihr begegnet man dem mittelbaren Einflusse der Digesten ${ }^{4}$ ). In Ansehung der Form bemerken wir, dass auch hier der Ausdruck „capitula" im Sinn von ,leges" wiederkehrt ${ }^{5}$ ), und dass in dieser Glossengruppe nicht selten am Anfange der Glossen die Wörter ,alias", ,id est" und „scilicet" fehlen, welche sich in den älteren Glossen gewöhnlich finden ${ }^{6}$ ).

Aus den vorstehenden Erörterungen geht, wie uns scheint, die Wahrheit unseres oben aufgestellten Satzes hervor, dass die Pistoieser Glossen nach ihrer Zeitfolge zusammengeordnet,

1) Glosse 16: Definition von metallum; Glosse 100: Definition von lex satura.

2) Glosse 16; Glosse 19: Definitionen von metus und vis.

3) Glosse 94.

$\left.{ }^{4}\right)$ Glosse 19. [Hier sclueint mir der unmittelbare Einfluss der Digesten (L. 1, 2, $3 \S 1$ D. quod met. c. 4, 2) ganz unverkennbar.

b) Glosse 11 .

Fitting.]

6) Siehe die Glossen 100, 148, 15̄5, 165, 173, 175, 176, 233, 238, $239,240,245,260,265,284,303,313,369,446,463,482,571,597,627$, $658,659,865,888,931,937$. 
in gleicher Weise wie die Turiner Institutionenglosse einen wichtigen Beitrag liefern zur Geschichte nicht nur der Erläuterung der Quellen, sondern überhaupt des Betriebes des römischen Rechtes während mehrerer Jahrhunderte des Mittelalters. Es zeigt sich, wic man von roh-ursprünglichen und alterthümlichen stufenweise $\mathrm{zu}$ mannigfaltigeren und ausgebildeteren Formen gelangt, wie allmählich der Gesichtskreis der Bearbeiter sich erweitert und neue Quellen von ihnen herangezogen werden.

Um zum Schlusse das Gesammtergebniss kurz zusammenzufassen, so umschliesst die Pistoieser Glosse eine Erläuterungsarbeit, welche, beginnend vor dem 10. und vielleicht sogar Fitting's Meinung gemäss vor dem 9 . Jahrhundert ${ }^{1}$ ), bis zum 13. Jahrhundert, in die glorreiche Zeit der Glossatoren fortgesetzt wird. Während sie auf der einen Seite mit der Rechtswissenschaft des Alterthums zusammenhängt und daher zu der Turiner Glosse und den Scholien der Epitome Iuliani Beziehungen hat, steht sie auf der anderen auch mit der juristischen Literatur des 10,, 11. und 12. Jahrhunderts in Verknüpfung, besonders mit dem Brachylogus, mit den Exceptiones des Petrus und ihrer Glosse, mit dem Libellus de Verbis legalibus und anderen alten Schriften, und erstreckt ihren Einfluss bis auf die Glosse des Accursins. Dass die Pistoieser Glosse wahren wissenschaftlichen Werth gehabt hat, zeigen auch die schon erwähnten Handschriften zu Darmstadt und zu Paris; worin sie zu einem Theil wiederkehrt, die eine dem 11., die andere dem 12. Jahrhundert angehörig. Sie weist alte Ueberlieferungen auf in den Scholien, in der Benutzung der lateinischen Uebersetzung der const. 4 Cod. VI, 4 mit Citaten in cler Form des Alterthums, endlich in der Beschaffenheit und der Fassung der Glossen; sie zeigt hinwieder Beziehungen zu der neuen Rechtsliteratur in den zahlreichen Definitionen, in der Benutzung der Digesten und der Werke des Isidor, in neuen Formen von Glossen und im Gebrauche einiger Ausdrücke, welche sich besonders in den Schriften nach 1000 finden. So ist sie ein neues Verbindungsglied zwischen dem früheren und dem späteren Mittelalter, ein

1) Vergl. Fitting in der mehrgedachten Anzeige S. 11. 
Dr. Schirmer, Replik in Sachen des linum testamenti incisum.

gewichtiger Beweis, dass in jenem Zeitalter die wissenschaftliche Ueberlieferung des römischen Rechtes niemals erloschen ist.

Pistoia, im Dezember 1886.

\title{
IV. \\ Replik in Sachen des linum testamenti incisum.
}

\author{
Von \\ Herrn Geheimerat Professor Dr. Schirmer \\ in Königsberg.
}

Die von mir in der ersten Abhandlung des siebenten Bandes dieser Zeitschrift gegen die fortdauernde civile Gültigkeit des Testamentes, dessen schliessendes Band durchschnitten ist, entwickelten Bedenken hat Krüger ebendaselbst Heft 3, p. 91-93 als völlig unberechtigt und hinfällig darzustellen versucht. Es mag mir vergönnt sein, hier kurz auseinanderzusetzen, weshalb seine Gründe mich in keiner Weise überzeugt haben.

Gegenüber dem Hinweis auf die Fragstellung in L. 20 D. de iniust. 28. 3. und das dadurch nahe gelegte argumentum a contrario, wonach bei nicht vorhandenem Wahnsinn des Erblassers auf eine abweichende Entscheidung des Juristen zu schliessen wäre, stellt Krüger die Behauptung auf, Scävola habe unverkennbar in seinen Digesten die Anfragen, abgesehen etwa von stilistischen Abweichungen, genau so geben wollen, wie sie ibm gestellt worden, und beschränke sich in den Antworten streng auf das ihm unterbreitete Material; die Entscheidung gebe er ohne Beifügung der Gründe und überlasse es den Lesern sich dieselben zurecht zu legen. Der erste Theil dieser Behauptung die unveränderte Widergabe der Anfragen betreffend, ist einmal, da diese im Original 